written

$$
T_{a b, \alpha \beta}(\nu)=\frac{1}{2}\left[t_{\alpha}, t_{\beta}\right]_{a b} T^{-}(\nu)+\frac{1}{2}\left\{t_{\alpha}, t_{\beta}\right\}_{a b} T^{+}(\nu)+\delta_{a b} \delta_{\alpha \beta} T^{0}(\nu),
$$

where $a$ and $b$ designate the isospin indices of the target pion in the initial and final states, respectively, and $\alpha$ and $\beta$ those of the incoming and scattered pion. $\left(t_{\alpha}\right)_{\beta \alpha}=-i \epsilon_{\alpha \beta \alpha} . T^{-}(\nu)$ is antisymmetric in $\nu$, while $T^{+}(\nu)$ and $T^{0}(\nu)$ are symmetric in $\nu$. Identifying the amplitudes in the above with the isospin amplitudes [G. F. Chew and S. Mandelstam, Phys. Rev. 119, $467(1960)$ ], we obtain

$$
T_{\pi^{-} \pi^{+}}=\frac{1}{2} T^{-}+\frac{1}{2} T^{+}+T^{0}, \quad T_{\pi^{+} \pi^{+}}=\frac{1}{2} T^{-}+\frac{1}{2} T^{+}+T^{0} .
$$

From these follow

$$
\operatorname{Im} \mathfrak{T}^{-}=2 \mu q\left[\sigma_{\pi^{-} \pi^{+}}-\sigma_{\pi^{+} \pi^{+}}\right]
$$

where $q$ is the pion momentum in the laboratory system.

${ }^{9}$ Without knowledge of the functional dependence of $\mathbb{M}^{-}$upon $k_{0}$, this continuation is not well defined. If one expresses the integral in the center-of-mass variable and continues in mass from $k_{0}=0$ to $k_{0}=\mu$, keeping energy $s$ fixed, under the assumption that all of the mass dependence is contained in the form factor, we expect for an arbitrary mass $k_{0}$

$$
\mathfrak{M}^{-}\left(s, k_{0}\right)=\approx\left[K_{\pi N N}\left(k_{0}\right)\right]^{2} \mathfrak{M}^{-}\left(s, k_{0}=\mu\right) .
$$

For further discussion of this continuation problem see reference 5 .

${ }^{10}$ L. M. Brown and P. Singer, Phys. Rev. 133 , B812 (1964).

${ }^{11}$ In the process of continuing the scattering amplitude in the pion mass (reference 9), there exists a certain ambiguity in the lower limit of integration in (11). If the physical pion mass $\mu$ is taken for this instead of $\frac{1}{2} \mu \sqrt{5}$ as in the case of the pion-pion forward-scattering dispersion integral, the value of the integral increases approximately by $10 \%$, resulting in $\left|G_{A} / G_{V}\right|=1.38$.

${ }^{12}$ S. C. Wojcicki, G. R. Kalbfleisch, and M. H. Alston, Phys. Letters $\underline{5}, 283$ (1963); D. H. Miller, G. Alexander, O. I. Dahl, L. Jacobs, G. R. Kalbfleisch, and G. A. Smith, Phys. Letters $\underline{5}, 279$ (1963).

\title{
SEARCH FOR A HEAVY ELECTRON BY ELECTRON-PROTON COINCIDENCE MEASUREMENTS
}

\author{
H. J. Behrend, F. W. Brasse, J. Engler, E. Ganssauge, and H. Hultschig \\ Deutsches Elektronen Synchrotron, Hamburg, Germany \\ and \\ S. Galster, G. Hartwig, and H. Schopper \\ Institut für Experimentelle Kernphysik des Kernforschungszentrums Karlsruhe \\ und der Technischen Hochschule Karlsruhe, Karlsruhe, Germany \\ (Received 25 October 1965)
}

Since measurements ${ }^{1}$ of electron-pair production at high momentum transfers indicate a deviation from quantum electrodynamics, the question of the existence of a heavy electron has been revived. Low ${ }^{2}$ suggested that an excited electron could be produced by the reaction $e+p \rightarrow e^{*}+p$ with the subsequent decay $e^{*} \rightarrow e+\gamma$. A search for such a particle has been carried out at Orsay ${ }^{3}$ by observing the recoil proton. A negative result was found for the mass range 240 to $570 \mathrm{MeV}$ (with $\lambda \lesssim 0.15$ at $570 \mathrm{MeV}$ ). Using electron-proton coincidences we have extended these measurements for masses $m_{e}$ * from 0.5 to $1.0 \mathrm{GeV}$. No evidence for a heavy electron was found. From the upper limits of the cross section, a value $\lambda \leqslant 0.02$ could be deduced where $\left(\lambda e / m_{e} e^{*}\right.$ is the coupling constant for the heavy electron.

Since the production process is a two-body reaction, the proton momentum is well defined for a given proton angle (and vice versa). Hence an excited electron would show up as a peak in the proton angular and momentum spectrum. The large background from inelastic processes (pion production and radiation losses) can be reduced almost completely for the major part of the kinematic region by measuring electronproton coincidences.

For the measurements an apparatus has been used which had been set up for the investigation of elastic $e-p$ scattering..$^{4}$ In a straight section of the synchrotron a liquid-hydrogen target is 
positioned which is viewed by two single-quadrupole spectrometers with a resolution of $5 \%$ full half-width. The solid angles of the proton and the electron arm were 5,6 and 4,6 msr, respectively. A shower counter was used in the electron spectrometer in order to distinquish electrons from pions. Only a crude $\pi$ proton discrimination by pulse-height selection was necessary in the proton spectrometer since $e-\pi$ coincidences are rejected almost completely by the overdetermined kinematics. Because of the geometrical limitations, the minimal angles are $32^{\circ}$ and $54.4^{\circ}$ for the electron and proton, respectively. In order to get a large cross section the production angle $\theta^{+}$of the heavy electron should be small. With consideration of the geometrical and other experimental limitations, the smallest possible production angle was chosen for each primary energy (see Fig. 1).

By varying the proton angle $\varphi_{p}$ and selecting the proper proton and electron momenta, different values of the heavy electron mass can be investigated. The proton angle was changed in steps of $0.5^{\circ}$ from $54.4^{\circ}$ to $62^{\circ}$. Since the spectrometer was touching the synchrotron magnet the measurement could not be extended to smaller angles. Since the acceptance of the spectrometer was $2^{\circ}$ a resonance should involve at least 3 points. Most of the measurements were performed at an incident electron energy of $4 \mathrm{GeV}$. The results are shown in Fig. 1. For the mass range $m_{e} e^{*}=500$ to 900 $\mathrm{MeV}$ the counting rates are practically zero. A steep rise was observed, however, for $m_{e}$ * $>900 \mathrm{MeV}$. In order to investigate this effect further measurements at 4.5 and $5.5 \mathrm{GeV}$ were carried out. The latter results are also displayed in Fig. 1. At $5.5 \mathrm{GeV}$ no rise in the counting rate was found. This shows that the steep rise at $4 \mathrm{GeV}$ cannot be attributed to a heavy electron. By additional measurements at 3.5 $\mathrm{GeV}$, it was possible to establish unambiguously that the observed counting rates correspond to the electroproduction of pions with intermediate isobar masses around $2.3 \mathrm{GeV}$. The steep rise in the $4-\mathrm{GeV}$ data is mainly due to a kinematical enhancement (solid curve in the figure). One approaches the maximum possible proton angle in the laboratory system where the ratio of the solid angle in the center of mass to that in the laboratory system becomes large. In addition there seems to be a rise of the counting rate due to the resonances near $2.2 \mathrm{GeV}$.

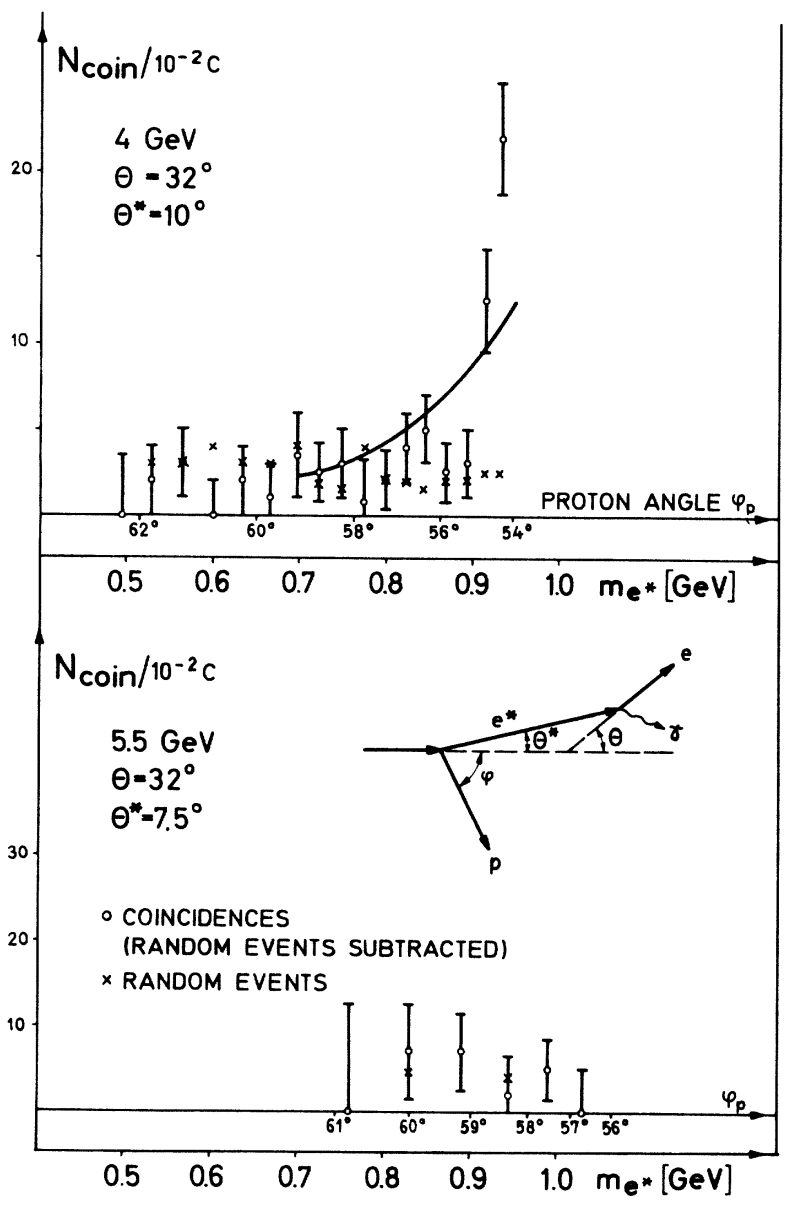

FIG. 1. The $e-p$ coincidence rate per quantameter charge as a function of the proton angle $\varphi_{p}$ for 4 and $5.5 \mathrm{GeV}$ primary electron energy. The constant of the quantameter [R. R. Wilson, Nucl. Instr. 1, 101 (1957)] was $4.85 \times 10^{18} \mathrm{MeV} / \mathrm{C}$. One $e-p$ coincidence per $10^{-2}$ C corresponds to a cross section of $2.05 \times 10^{-34} \mathrm{~cm}^{2} /$ $\mathrm{sr}^{2}$. Each proton angle corresponds to a certain mass $m_{e}{ }^{*}$ of the heavy electron which is also indicated in the figure. The solid curve has been calculated for the competing electroproduction of single pions taking into account the phase-space factor only.

A detailed account of the electroproduction measurements will be reported elsewhere..$^{5,6}$

From the observed counting rates upper limits of the cross sections for the production of heavy electrons can be deduced. They are shown in Table I. If these experimental results are compared to theoretical cross sections $\mathbf{s}^{3,7}$ computed with the coupling suggested by Low, ${ }^{2}$ one can infer upper limits for the coupling constant $\lambda .^{8}$ Our results are compatible with $\lambda^{2}$ $\varsigma 4 \times 10^{-4}$ whereas $\lambda^{2} \sim 1$ could be expected theoretically. Hence a heavy electron with a mass 
Table I. Upper limits on the experimental cross section for the production of a heavy electron with mass $m_{e}{ }^{*}$. The theoretical cross section and lower limits on $\lambda$ are also shown.

\begin{tabular}{|c|c|c|c|c|}
\hline$m_{e}{ }^{*}[\mathrm{MeV}]$ & $\begin{array}{c}\left(\partial^{2} \sigma / \partial \Omega_{e} e^{\mathrm{Lab}} \partial \Omega_{p}{ }^{\mathrm{Lab}}\right) \exp \\
\left(10^{-33} \mathrm{~cm}^{2} / \mathrm{sr}^{2}\right)\end{array}$ & $\begin{array}{l}\left(\partial \sigma / \partial \Omega e^{*}{ }^{\mathrm{cm}}\right) \exp \\
\left(10^{-33} \mathrm{~cm}^{2} / \mathrm{sr}\right)\end{array}$ & $\begin{array}{l}\left(\partial \sigma / \partial \Omega e^{*}{ }^{\mathrm{cm}}\right) \text { theo } \\
\left(10^{-30} \mathrm{~cm}^{2} / \mathrm{sr}\right)\end{array}$ & $\lambda \times 10^{2}$ \\
\hline 500 & 0.41 & 1.53 & 7.44 & $\lesssim 1.4$ \\
\hline 600 & 0.41 & 1.07 & 5.32 & $\leqslant 1.4$ \\
\hline 700 & 0.41 & 0.80 & 3.52 & $\lesssim 1.5$ \\
\hline 800 & 0.41 & 0.65 & 2.48 & $\leqslant 1.7$ \\
\hline 900 & 0.41 & 0.52 & 1.84 & $\lesssim 1.7$ \\
\hline 1000 & 1.10 & 1.59 & 2.60 & $\lesssim 2.5$ \\
\hline
\end{tabular}

between 0.2 and $1 \mathrm{GeV}$ does not exist. The limits on $\lambda$ also imply that the pair production results must be explained in a different way. ${ }^{7,9}$

We should like to thank Dr. F. Gutbrod, Dr. H. Joos, and Dr. W. Schmidt for many illuminating discussions and their help in computing the kinematics and the cross sections. We gratefully acknowledge the help of Dr. Pingel in constructing the hydrogen target. The cooperation of the synchrotron staff is also appreciated.

${ }^{1}$ R. B. Blumenthal, D. C. Ehn, W. L. Faissler, P. M. Joseph, L. J. Lanzerotti, F. M. Pipkin, and D. G. Stairs, Phys. Rev. Letters 14, 660 (1965).

${ }^{2}$ F. E. Low, Phys. Rev. Letters 14, 238 (1965).

${ }^{3} \mathrm{C}$. Bétourné, H. Nguyen Ngoc, J. Perez y Jorba, and J. Tran Thanh Van, Phys. Letters 17, 70 (1965).

${ }^{4}$ H. J. Behrend, F. W. Brasse, J. Engler, E. Ganssauge, and H. Hultschig, Deutsches Elektronen Synchrotron Report No. DESY 65-3; and S. Galster, G. Hart- wig, and H. Schopper, Kernforschungszentrums Karlsruhe Report No. KFK 320.

${ }^{5}$ H. J. Behrend, F. W. Brasse, J. Engler, E. Ganssauge, and H. Hultschig, Deutsches Elektronen Synchrotron Report No. DESY 65-9; and S. Galster, G. Hartwig, and H. Schopper, Kernforschungszentrums Karlsruhe Report No. KFK 362.

${ }^{6}$ Here we want only to mention that if one converts our data on the differential cross section $d^{3} \sigma / d \Omega_{e}$ $\times d \Omega_{p} d P_{p}$ into values for photoproduction or total electroproduction by making plausible assumptions, one obtains cross sections which are about 25 times larger than those published in the literature: H. H. Cone. K. W. Chen, J. R. Dunning, Jr., G. Hartwig, N. F. Ramsey, G. K. Walker, and R. Wilson, Phys. Rev. Letters 14, 326 (1965); R. Alvarez, Z. Bar Yam, W. Kern, D. Luckey, L. S. Osborne, S. Tazzari, and R. Fessel, Phys. Rev. Letters 12, 707 (1964).

${ }^{7}$ F。 Gutbrod and D. Schildknecht, Deutsches Elektronen Synchrotron Report No. DESY 65-10.

${ }^{8}$ Note that our $\lambda$ differs from that introduced by Low in the following way: $\lambda_{\text {Low }}=\lambda e / m_{e}{ }^{*}$.

${ }^{9} \mathrm{~F}$. Gutbrod and H. Joos, private communication.

\title{
RENORMALIZATION EFFECTS AND THE CABIBBO ANGLE
}

\author{
H. T. Nieh*
}

Department of Physics, Harvard University, Cambridge, Massachusetts (Received 11 October 1965)

We report in this note calculations of the renormalization of the semileptonic weak coupling constants by the $\mathrm{U}(3)$ symmetry-breaking interactions. Our consideration is based on a phenomenological field-theoretical model of $\mathrm{U}(3)$ symmetry breakdown, which is an extension of the idea that the symmetry breakdown is dominated by the nonvanishing expectation value of the scalar field $S_{33} \cdot{ }^{1,2}$ The conclusions of this paper depend essentially on meson-pole dominance approximations. Details and other related results will be submitted for publica- tion elsewhere.

In this model, we assume that the phenomenological Lagrange function for the $0^{-}$meson fields $\Phi_{a b}, 1^{-}$meson fields $U_{a b}$, and $\frac{1}{2}^{+}$baryon fields $\Psi_{a b}$ is of the form

$$
\mathfrak{L}=\mathfrak{L}_{0}+\mathscr{L}_{\text {int }} \mathfrak{L}_{33},
$$

where $\mathscr{L}_{0}$ is the free-field Lagrange function, ${ }^{3}$ $\mathcal{L}_{\text {int }}$ represents the U(3)-symmetric strong interactions, and $\mathscr{L}_{33}$ effectively represents the symmetry-breaking effects resulting from the 